\title{
Trombólisis con activador recombinante del plasminógeno tisular (rt-PA) para el ataque cerebro vascular agudo: la experiencia colombiana
}

\author{
Thrombolysis with rt-PA for acute stroke: Colombian experience
}

\section{David A. Pineda (1)}

\section{RESUMEN}

El tratamiento con activador recombinante del plasminógeno tisular (sigla en inglés rt-PA), aplicado por vía intravenosa (VIV) es el procedimiento de primera línea en casos de ataque cerebrovascular agudo (ACVA) en una ventana de 4,5 horas. En Colombia hay una experiencia publicada del uso exitoso de este medicamento. En el número actual se publica una nueva experiencia del uso de rt-PA en el caribe colombiano, de gran utilidad para reseñar los artículos publicados en Colombia acerca del tema.

PALABRAS CLAVES: trombolisis, ataque cerebrovascular agudo, ACV, rt-PA, alteplase.

\begin{abstract}
Treatment with intravenous recombinant tissue plasminogen activator (rt-PA) for acute stroke (AS) is the first line procedure for patients in 4,5 hours window. In Colombia there is a published experience about the successful use of this medication. In the currently issue a new experience from Colombian Caribbean is presented, which it is taken in advantage for briefly reviewing the published papers about the theme in our country.
\end{abstract}

KEY WORDS: thrombolysis, Acute Stroke, Stroke, rt-PA, Alteplase.

La utilidad del activador recombinante del plasminógeno tisular (sigla en inglés rt-PA), aplicado por vía intravenosa (VIV), para el tratamiento del ataque cerebrovascular agudo (ACVA), ha sido plenamente establecida para pacientes en la ventana terapéutica de 4,5 horas, a partir del inicio de los síntomas de ACVA y con bajo riesgo de transformación hemorrágica del infarto cerebral (1-3). Incluso, en un metaanálisis reciente, este efecto benéfico se mantiene a pesar de la edad, superior a 80 años y de forma independiente del tamaño del infarto cerebral (1).

En Colombia existe un consenso nacional entre diversos especialistas médicos, apoyado por el Ministerio de Protección Social para el uso del rt-PA por VIV para la ventana de 4,5 horas, o por vía intra-arterial (VIA) para la ventana de 6 horas, en casos de ACVA (4), el cual debería ser seguido por los centros de urgencias, a los cuales accedan los pacientes.
Se sugiere el uso de la escala NIHSS para evaluar la severidad clínica y la escala cuantitativa del tamaño de la lesión cerebral en el TAC (sigla en inglés: ASPECTS). El procedimiento debe realizarse en entidades de referencia; el paciente debe tener la evaluación previa de un neurólogo, quien debe constatar los criterios de inclusión del paciente para prescribir el procedimiento y descartar todos los criterios de exclusión establecidos por el consenso, de acuerdo con los estudios de clase I y con un nivel de evidencia A.

Se han publicado varios artículos acerca del uso de rt-PA en Colombia, uno de ellos hace referencia a la elaboración de la guía médica de trombolisis en Colombia, que presenta un análisis de la comparación de costo/efectividad del tratamiento tradicional de soporte del ACVA versus el uso de rt-PA (5), el cual concluyó que, pese al mayor costo inicial de esta última intervención, los beneficios a largo plazo,

(1) MD, PhD (h.c.). Profesor titular de Neurología y Neuropsicología. Universidad de San Buenaventura, Medellín, Colombia. Universidad de Antioquia, Medellín, Colombia.

Recibido: 22/3/17. Aceptado: 22/3/17.

Correspondencia: David A. Pineda, david.pineda.editor@acnweb.org 
calculados de acuerdo con la medición de años de vida ajustados por su calidad (sigla en inglés QUALY), muestran que por cada año se debe invertir $\$ 1.138 .373$ adicionales en los pacientes que recibieron la terapia tradicional.

Otro estudio realizado en 24 pacientes con ACVA, de edades comprendidas entre 65 y 79 años, que recibieron rt-PA VIV, se encontró que en el grupo predominaron las mujeres (79 \%), el mayor factor de riesgo fue la hipertensión arterial (HTA) (18/24), seguido de la fibrilación auricular (FA) $(7 / 24)$ y el tabaquismo (6/24). Al momento de la intervención, el $62,5 \%$ de los pacientes tenían una escala de NIHSS entre 15 y 24 . El $58 \%$ de los pacientes tuvieron una puntuación de 0 a 2 en la escala Rankin modificada a los 3 meses (6).

En una investigación realizada a 15 pacientes con ACVA en Cali, que consultaron al servicio de urgencias de una clínica de alto nivel de atención, dentro de la ventana terapéutica de 4,5 horas, fueron tratados con rt-PA VIV. En la muestra predominaron los hombres $(60 \%)$, la edad promedio fue de $68,7 \pm 8,8$ años, todos los pacientes ingresaron con hemiparesia, con una escala de NIHSS previa al procedimiento de $12,9 \pm 4,9$. El factor de riesgo más frecuente fue la HTA (80 \%), seguido por la diabetes (53,3\%). La principal complicación inmediata a la intervención fue la transformación hemorrágica en el $20 \%$ de los casos (7).
Recientemente se hizo una revisión crítica (8) para comparar el uso de rt-PA VIV versus la terapia endovascular (TEV). A pesar de encontrarse beneficios en el uso de la TEV en pacientes que están por fuera de la ventana terapéutica, se evidenciaron serias limitaciones para su uso en Colombia por razones de disponibilidad tecnológica.

En el número actual de Acta Neurológica Colombiana (Vol. 33, núm. 1), se presenta una nueva experiencia del uso de la trombolisis con rt-PA VIV en 27 pacientes de la ciudad de Barranquilla con ACVA, la edad promedio de estos pacientes fue de 65 años, el principal factor de riesgo fue la HTA (19/27). Se encontró que más del $50 \%$ de los pacientes tuvieron un escalafón de 0 a 1 a los 3 meses, lo que indica un desenlace muy favorable en esta muestra (9).

Estas experiencias del uso del rt-PA en Colombia deberían motivar a los neurólogos dedicados al tema de la ACVA a entrar en los grupos de protocolos internacionales (10), para la ejecución de meta-análisis interinstitucionales, que permitan mejorar los desenlaces clínicos, mediante la ejecución de procedimientos estandarizados, para detectar y tratar a los pacientes con ACVA de manera oportuna.

\section{Conflicto de intereses}

Los autores manifiestan no tener conflictos de intereses en este estudio.

\section{REFERENCIAS}

1. Hacke W, Donnan G, Fieschi C, Kaste M, von Kummer R, Broderick JP, et al. Association of outcome with early stroke treatment: pooled analysis of ATLANTIS, ECASS, and NINDS rt-PA stroke trials. Lancet. 2004;363(9411): 768-74.

2. Lees KR, Bluhmki E, von Kummer R, Brott TG, Toni D, Grotta JC, et al. Time to treatment with intravenous alteplase and outcome in stroke: an updated pooled analysis of ECASS, ATLANTIS, NINDS, and EPITHET trials. Lancet. 2010;375(9727):1695-703. http://dx.doi.org/10.1016/S01406736(10)60491-6.

3. Emberson J, Lees KR, Lyden P, Blackwell L, Albers G, Bluhmki E, et al. Effect of treatment delay, age, and stroke severity on the effects of intravenous thrombolysis with alteplase for acute ischaemic stroke: a meta-analysis of individual patient data from randomised trials. Lancet. 2014;384(9958):1929-35. http:// dx.doi.org/10.1016/S0140-6736(14)60584-5.

4. Red Nacional de Trombolisis. Consenso Nacional de Trombolisis y Terapias de Reperfusión. Ministerio de Protección Social. Grupo de Atención de Emergencias y Desastres. Bogotá DC. 2008.

5. Muñoz-Collazos M, Gutiérrez AM, Londoño D, Bayona $\mathrm{H}$, Herrán S, Pérez GE. Uso del Activador de Plasminógeno Tisular Recombinante (rt-PA) en el Ataque Cerebrovascular isquémico
(ACVi) en Colombia: un estudio de costo-efectividad. Acta Neurol Colomb. 2008;24(4):158-73.

6. Castañeda-Cardona C, Coral-Casas J, Rueda MC, Díaz-Cortés D, Ruiz A. Experiencia de trombólisis intravenosa en el manejo del ataque cerebro vascular en el Hospital Universitario San Ignacio 2011-2013 (EXTRO HUSI). Acta Neurol Colomb. 2014;30(1):16-21.

7. Guerrero-González Luis Fernando, Serna JJ, Gámez DX. Experiencia de trombolisis intravenosa en infarto cerebral agudo en un hospital universitario de Cali. Acta Med Colomb. 2014;39(2, Supl.1):46.

8. Torres-Zafra J, Fonnegra-Caballero A, Vicini-Parra J. Terapia endovascular en el tratamiento del ACV isquémico agudo. Perspectiva en Colombia. Acta Neurol Colomb. 2015;31(3):335-41.

9. Hernández-Ruiz EA, Guarín-Navas EG, Lora-Acuña FJ, Acosta-Reyes J, Beltrán-Carrascal E, Meza Cely N. Acta Neurol Colomb. 2017;33(1):275-84.

10. The Stroke Thrombolysis Trialists' Collaborative Group. Details of a prospective protocol for a collaborative meta-analysis of individual participant data from all randomized trials of intravenous rt-PA vs. control: statistical analysis plan for the Stroke Thrombolysis Trialists' Collaborative meta-analysis. Int J Stroke. 2013;8(4):278-83. http://dx.doi.org/10.1111/ijs.12040 